\title{
High-energy neutrino-nucleus interactions
}

\author{
Shunzo Kumano ${ }^{1,2, *}$ \\ ${ }^{1}$ KEK Theory Center, Institute of Particle and Nuclear Studies, KEK, \\ 1-1, Ooho, Tsukuba, Ibaraki, 305-0801, Japan \\ ${ }^{2}$ J-PARC Branch, KEK Theory Center, Institute of Particle and Nuclear Studies, KEK, \\ and Theory Group, Particle and Nuclear Physics Division, J-PARC Center, \\ 203-1, Shirakata, Tokai, Ibaraki, 319-1106, Japan
}

\begin{abstract}
High-energy neutrino-nucleus interactions are discussed by considering neutrino-oscillation experiments and ultra-high-energy cosmic neutrino interactions. The largest systematic error for the current neutrino oscillation measurements comes from the neutrino-nucleus interaction part, and its accurate understanding is essential for high-precision neutrino physics, namely for studying CP violation in the lepton sector. Depending on neutrino beam energies, quasi-elastic, resonance, Regge, or/and deep inelastic scattering (DIS) processes contribute to the neutrino cross section. It is desirable to have a code to calculate the neutrino-nucleus cross section in any kinematical range by combining various theoretical descriptions. On the other hand, the IceCube collaboration started obtaining cross section data up to the $10^{15} \mathrm{eV}$ range, so that it became necessary to understand ultra-high-energy neutrino interactions beyond the artificial lepton-accelerator energy range. For future precise neutrino physics including the $\mathrm{CP}$ measurement, it is also necessary to understand accurate nuclear corrections. The current status is explained for nuclear corrections in DIS structure functions. The possibility is also discussed to find gravitational sources within nucleons and nuclei, namely matrix elements of quark-gluon energy-momentum tensor. They could be probed by neutrino interactions without replying on direct ultra-weak "gravitational interactions" with high-intensity neutrino beams, possibly at a future neutrino factory, by using techniques of hadron tomography.
\end{abstract}

\section{Introduction}

In recent years, precise descriptions of high-energy neutrino-nucleon and neutrino-nucleus reactions became necessary. First, it is motivated by the progress of neutrino oscillation experiments. The measurements are getting more and more precise, and it became the stage of probing $\mathrm{CP}$ violation in leptons. The target is water, for example, in the $\mathrm{T} 2 \mathrm{~K}$ experiment, so that accurate neutrino-oxygen cross sections should be calculated in addition to neutrinonucleon ones [1]. Roughly, an accuracy of 5\% is required for the theoretical estimate on the neutrino-nucleus cross sections for future neutrino-oscillation measurements.

Second, ultra-high-energy neutrino experiments have been done recently by the IceCube collaboration. In fact, the first cross section measurement was reported in the $10^{13}-10^{15} \mathrm{eV}$ range by the IceCube in 2017 [2]. So far, the IceCube cross sections are consistent with the standard model estimate within their errors. In the near future, we expect to have much experimental progress in this field by considering other future plans on high-energy neutrino experiments such as KM3NeT (Cubic Kilometer Neutrino Telescope) and Baikal GVD (Gigaton Volume Detector).

Considering these circumstances, we explain descriptions of high-energy neutrino-nucleon and neutrinonucleus cross sections by focusing mainly on the deep-

\footnotetext{
*e-mail: shunzo.kumano@kek.jp
}

inelastic-scattering (DIS) part. The current status and future prospect of structure functions and parton distribution functions are summarized in Ref. [3]. In Sec. 2, an outline of neutrino-interaction descriptions is discussed, and the details are explained for neutrino DIS processes in Sec.3, together with nuclear corrections. The current status of cosmic ultra-high-energy neutrino cross sections is explained in Sec. 3.2. Nuclear modifications of the structure function $F_{2}$ and the PDFs are shown in Sec.3.3. It is possible to probe gravitational form factors of hadrons by neutrino scattering as explained in Sec. 4. The current situation for determining the gravitational form factors from the KEKB measurements is also shown. These discussions are summarized in Sec. 5.

\section{Neutrino-nucleus interactions}

Kinematical regions of neutrino-nucleus scattering are shown in Fig. 1 by considering the neutrino energies from $\mathrm{MeV}$ to multi $\mathrm{GeV}$, namely current beam energies of neutrino oscillation experiments. There are four kinematical regions as shown in Fig. 1: quasi-elastic (QE), resonance (RES), deep inelastic scattering (DIS), and Regge (REG). Here, four-momentum and energy transfers are denoted $q$ and $v$, respectively, and $Q^{2}$ is defined by $Q^{2}=-q^{2}$.

The elastic scattering occurs if the relation $v=$ $Q^{2} /\left(2 M_{A}\right)$, where $M_{A}$ is the nuclear mass, is satisfied. At 


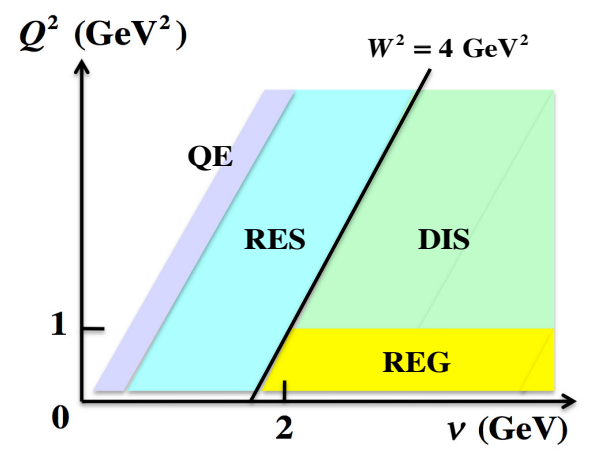

Figure 1. Kinematical regions of neutrino-nucleus scattering.

low energy neutrino scattering, this relation is given approximately by $v \simeq \vec{q}^{2} /\left(2 M_{A}\right)$. It means that the energy transfer is equal to the recoil energy of a nucleus. In this elastic scattering, the neutrino sees the nucleus as a whole system. As the neutrino energy increases, the Compton wavelength becomes short enough to resolve individual nucleons in the nucleus. If the nucleon is at rest, the kinematical relation becomes $v=Q^{2} /\left(2 M_{N}\right) \simeq \vec{q}^{2} /\left(2 M_{N}\right)$. Then, we take into account binding and Fermi-motion effects. The binding effect could be effectively included into a modified nucleon mass $M_{N}^{*}$ as a rough estimate, so that the kinematical relation is expressed by the nucleon momentum $\vec{p}$ as $v \simeq(\vec{p}+\vec{q})^{2} /\left(2 M_{N}^{*}\right)-\vec{p}^{2} /\left(2 M_{N}^{*}\right)$. By using the nucleon's Fermi momentum $p_{F}$, it is expressed as $\vec{q}^{2}-2 q p_{F} \leq 2 M_{N}^{*} v \leq \vec{q}^{2}+2 q p_{F}$ [4]. This is the quasielastic region shown in Fig. 1.

As the neutrino energy increases further, the nucleon is excited into resonances, and this kinematical region is given by the invariant-mass squared as $M_{N}^{2}<W^{2}=$ $(p+q)^{2}<4 \mathrm{GeV}^{2}$. This is the resonance region. At $W^{2}=(p+q)^{2} \geq 4 \mathrm{GeV}^{2}$ and $Q^{2} \geq 1 \mathrm{GeV}^{2}$, the nucleon is broken up into hadron pieces, and it is the deepinelastic-scattering (DIS) region. The neutrino scattering in this region is described by partons within the nucleus. Because the photon interacts with the partons within a short time, interactions among the partons could be neglected for describing the process. Namely, the reaction is described by the incoherent impulse approximation from individual partons. For the parton description with the incoherent assumption, the $Q^{2}$ value should be large enough. Furthermore, $Q^{2}$ dependencies of structure functions or parton distribution functions (PDFs) are described by the Dokshitzer-Gribov-Lipatov-Altarelli-Parisi (DGLAP) evolution equations [5] in the perturbative QCD region. The running coupling constant $\alpha_{s}\left(Q^{2}\right)$ should be small enough for the perturbative calculations, and it means $Q^{2}$ is large enough: $Q^{2} \geq 1 \mathrm{GeV}^{2}$ or a few $\mathrm{GeV}^{2}$. There is another region at $W^{2} \geq 4 \mathrm{GeV}^{2}$ and $Q^{2}<1$ $\mathrm{GeV}^{2}$, and the reaction is described by the Regge theory with Reggeons and Pomerons. There are also guidelines from the quark-hadron duality and the partially conserved axial current (PCAC) in the limit $Q^{2} \rightarrow 0$ for describing the axial-vector current part, whereas the vector part is conserved.

In Fig. 2, each contribution is shown as a function of neutrino energy [6]. At low energies with $E_{v}<1 \mathrm{GeV}$

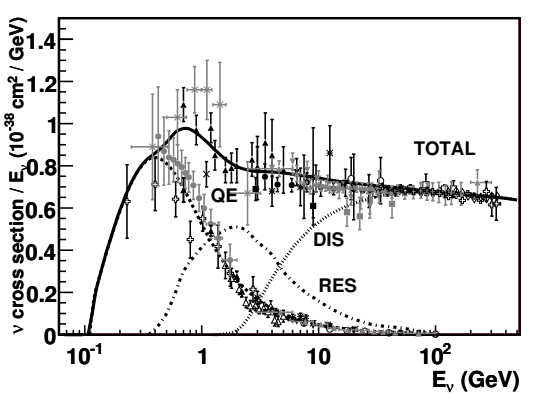

Figure 2. Neutrino scattering cross sections [6] .

such as $\mathrm{T} 2 \mathrm{~K}$, the reactions are dominated by the quasielastic scattering and there are some resonance effects. On the other hand, the DIS processes started to contribute in the multi-GeV region of Fermilab neutrino energies. All the different kinematical regions need to be understood precisely for neutrino-oscillation measurements, and the current situation is summarized in Ref. [1]. The ultra-high energy cosmic neutrino reactions are dominated by the DIS in general, except for the forward scattering. However, the energy range is beyond our current experimental understanding by lepton accelerators, so that theoretical extrapolations are needed for calculating neutrino cross sections at very high energies.

\section{Deep inelastic neutrino-nucleus scattering}

\subsection{Neutrino cross sections and structure functions}

In this article, we do not step into the low-energy descriptions of neutrino-nucleus interactions. One may look at the summary article [1] for such information. Here, we focus on the DIS part. The charged-current (CC) cross section for neutrino (or antineutrino) proton scattering is expressed by three structure functions, $F_{1}$ (or $F_{L}$ ), $F_{2}$, and $F_{3}$ as [7]

$$
\begin{aligned}
& \frac{d \sigma_{C C}^{v / \bar{v}}}{d x d y}=\frac{G_{F}^{2} s}{2 \pi\left(1+Q^{2} / M_{W}^{2}\right)^{2}}\left[F_{1}^{C C} x y^{2}\right. \\
& \left.+F_{2}^{C C}\left(1-y-\frac{M x y}{2 E}\right) \pm F_{3}^{C C} x y\left(1-\frac{y}{2}\right)\right]
\end{aligned}
$$

where $x$ is the Bjorken scaling variable defined by $x=Q^{2} /(2 p \cdot q), \pm$ indicates + and - for neutrino and antineutrino, respectively, $G_{F}$ is the Fermi coupling constant, $y$ is defined by $y=p \cdot q / p \cdot k=v / E$ with the neutrino momentum $k$ and energy $E, M_{W}$ is the $W$ boson mass, and $s$ is the center-of-mass energy. In parton model, the structure function $F_{1}$ is related to $F_{2}$ by the Callan-Gross relation $2 x F_{1}^{C C}=F_{2}^{C C}$, and the structure functions are expressed by the PDFs for the proton as

$$
\begin{aligned}
F_{2}^{v p(C C)} & =2 x(d+s+\bar{u}+\bar{c}), \\
x F_{3}^{v p(C C)} & =2 x(d+s-\bar{u}-\bar{c}), \\
F_{2}^{\bar{v} p(C C)} & =2 x(u+c+\bar{d}+\bar{s}), \\
x F_{3}^{\bar{v} p(C C)} & =2 x(u+c-\bar{d}-\bar{s}) .
\end{aligned}
$$


On the other hand, the neutral-current (NC) cross sections are expressed in the similar way as

$$
\begin{aligned}
& \frac{d \sigma_{N C}^{v / \bar{v} p}}{d x d y}=\frac{\rho G_{F}^{2} s}{2 \pi\left(1+Q^{2} / M_{Z}^{2}\right)^{2}}\left[F_{1}^{N C} x y^{2}\right. \\
& \left.+F_{2}^{N C}\left(1-y-\frac{M x y}{2 E}\right) \pm F_{3}^{N C} x y\left(1-\frac{y}{2}\right)\right]
\end{aligned}
$$

where $M_{Z}$ is the Z-boson mass, $\sin \theta_{W}$ is the weak-mixing angle, and $\rho=M_{W}^{2} /\left(M_{Z}^{2} \cos ^{2} \theta_{W}\right)$. In the parton model, $F_{1}$ is related to $F_{2}$ as $2 x F_{1}^{N C}=F_{2}^{N C}$, and the proton structure functions are expressed by the PDFs as

$$
\begin{gathered}
F_{2}^{v / \bar{v} p(N C)}=2 x\left[\left(u_{L}^{2}+u_{R}^{2}\right)\left(u^{+}+c^{+}\right)+\left(d_{L}^{2}+d_{R}^{2}\right)\left(d^{+}+s^{+}\right)\right], \\
x F_{3}^{v / \bar{v} p(N C)}=2 x\left[\left(u_{L}^{2}-u_{R}^{2}\right)\left(u^{-}+c^{-}\right)+\left(d_{L}^{2}-d_{R}^{2}\right)\left(d^{-}+s^{-}\right)\right],
\end{gathered}
$$

where $q^{ \pm}$is defined by $q^{ \pm} \equiv q \pm \bar{q}$. The left- and right-hand couplings for a quark are expressed by the third component of isospin $T_{a}^{3}$, charge $e_{q}$, and the weak-mixing angle $\theta_{W}$ as

$$
\begin{gathered}
q_{L}=T_{q}^{3}-e_{q} \sin ^{2} \theta_{W}, q_{R}=-e_{q} \sin ^{2} \theta_{W}, \\
T_{q}^{3}, e_{q}=\left\{\begin{array}{ll}
+1 / 2,+2 / 3 & \text { for } q=(u, c) \\
-1 / 2,-1 / 3 & \text { for } q=(d, s)
\end{array} .\right.
\end{gathered}
$$

The parton-model expressions are given in the leading order of the running coupling constant $\alpha_{s}$. Higher-order corrections are included in coefficient functions together with the gluon distribution.

\subsection{Ultra-high-energy cosmic neutrino interactions}

The structure functions have been measured mainly in charged-lepton DIS processes for nucleons and nuclei. There are also experiments in neutrino DIS particularly by the NuTeV collaboration. The highest-energy measurements by artificial accelerators were done at HERA (Hadron-Electron Ring Accelerator) for the nucleon structure functions. However, there are ultra-high-energy cosmic neutrinos reaching the earth and are detected by the IceCube facility in the antarctic. The ultra-high-energy neutrino reactions provide valuable information on nucleon structure studies in the energy region beyond the current artificial accelerators, new physics beyond the standard model, astrophysical neutrino production, and propagation in the cosmic microwave background, and the Greisen-Zatsepin-Kuzmin (GZK) mechanism.

Ultra-high-energy comic neutrino measurements have been done by the IceCube collaboration. Recently, there was a first report on neutrino cross sections [2]. Cosmic neutrinos pass through the earth's interior and interact with the ice surrounding the IceCube detectors in the ice cap at 1,450-2,450 meters below its surface. Their results are shown in Fig. 3 for the charged-current (CC) cross sections in the energy range of $10^{13}-10^{15} \mathrm{eV}$ together with artificial accelerator measurements at lower energies, separately, for neutrinos and antineutrinos. The neutrino CC interactions in the ice create charged muons, which are detected as long tracks of Cherenkov light. However, they

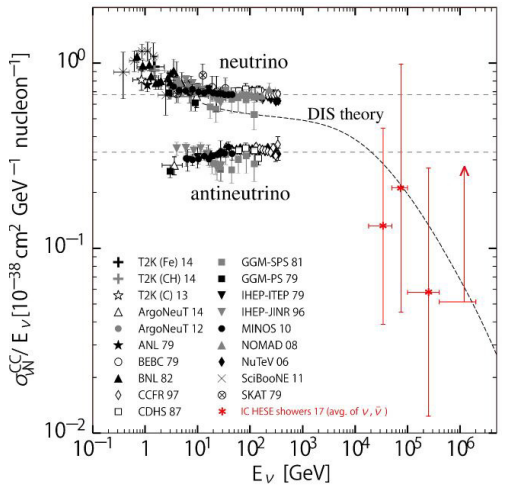

Figure 3. Ultra-high-energy neutrino cross sections [2] (Figure supplied by M. Bustamante).

are not able to distinguish neutrino events from antineutrino ones and they are also not able to detect neutral current events. In this way, the $\mathrm{CC}$ cross sections were obtained in the IceCube experiment as shown in Fig. 3.

The DIS curve is calculated from the theoretical formalism of Ref. [7] by assuming the same mixture of neutrino and antineutrino events and by extending our knowledge of the accelerator-based PDFs to the higher-energy region. Within the errors of the IceCube measurements, the standard model curve is consistent with the data. In future, more accurate measurements are expected from the IceCube, and there are also future projects of $\mathrm{KM} 3 \mathrm{NeT}$ and Baikal-GVD on ultra-high-energy cosmic neutrino measurements, so that we should keep our eyes on these measurements. Since they are in the energy region beyond our accelerators, there may be new phenomena and new physics beyond the current standard model.

\subsection{Nuclear modifications}

As neutrino measurements become accurate in the neutrino oscillation experiments, it became necessary to understand nuclear corrections because neutrino-oxygen interactions are involved in the measurements, although they are partly constrained by near-detector experiments. For future CP violation measurements for the lepton sector, neutrino-nucleus cross sections should be calculated within about $5 \%$ accuracy. The corrections are generally 20-30\% effects in structure functions and the PDFs for medium and large nuclei. They need to be taken into account for a precise cross section estimate.

Now, nuclear modifications of the structure function $F_{2}$ are relatively well known for nuclei with small mass numbers to large ones in charged-lepton DIS, although the small $x(<0.004)$ region are yet to be investigated by the Electron-Ion Collider (EIC) project. The typical nuclear modification measurements are shown in Fig. 4 for the carbon nucleus as a function of the scaling variable $x$. The open squares indicate the data with small invariant mass $W^{2}<4 \mathrm{GeV}^{2}$, so they are not DIS data to be precise. However, the rise of the large- $x$ ratio agrees with theoretical expectation based on a convolution model with nucleon Fermi motion effects, so that they are shown together with the DIS data to see the general $x$-dependence tendency.

At small $x(<0.05)$, the modifications are negative and they are caused by nuclear shadowing. The virtual 


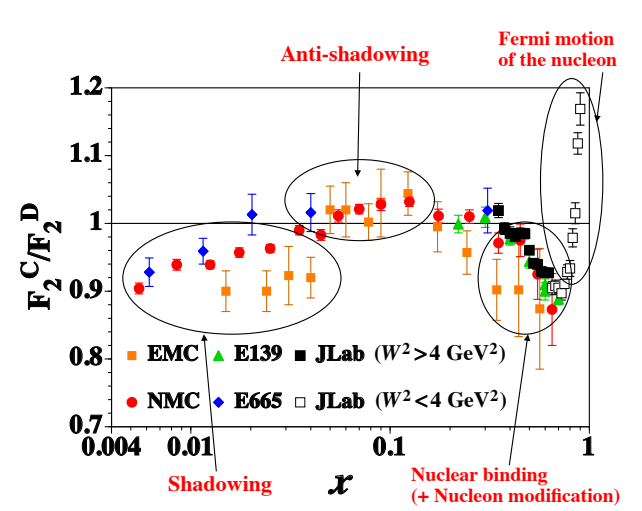

Figure 4. Nuclear modifications in $F_{2}^{C} / F_{2}^{D}$.

photon could fluctuate into a $q \bar{q}$ or a vector meson state at small $x$. The $q \bar{q}$ propagation length is estimated as $\lambda=1 /\left|E_{V}-E_{\gamma}\right|=2 v /\left(Q^{2}+M_{V}^{2}\right)=0.2 \mathrm{fm} / x>2 \mathrm{fm}$ at $x<0.1$. It becomes larger than the average separation of nucleons in a nucleus, and multiple scattering occurs. A double-scattering contribution is negative to the single scattering one, which results in the shadowing. Namely, the $q \bar{q}$ pair or a vector meson strongly interacts with surface nucleons, so that the projectile does not interact with internal nucleons and they are shadowed by the surface ones.

The medium- and large- $x$ regions $(x>0.3)$ of the nuclear modifications are generally described by the convolution picture. Namely, the nuclear structure function $F_{2}^{A}$ is calculated by the convolution integral of the nucleonic structure function $F_{2}^{N}$ with the spectral function, which indicates the nucleon momentum distribution in a nucleus. The spectral function contains nuclear binding and nucleon Fermi-motion effects. The nuclear binding and Fermi motion are of the order of $10-100 \mathrm{MeV}$ range, which is very small in comparison with the DIS energies of $10-100 \mathrm{GeV}$. However, a slight shift in the nucleon lightcone-momentum distribution results in the $10 \%$ modifications in the carbon nucleus through the structure function $F_{2}^{N}$. The Fermi-motion increase with $x$ at large $x$ $(>0.8)$ is also understood in the same theoretical framework of the convolution description. Finite nuclear structure functions $F_{2}^{A}$ exist in the kinematical region $0<x<A$ where $A$ is the mass number, whereas it should vanish for the nucleon at $x=1$. It means that the ratio becomes $F_{2}^{A} / F_{2}^{N} \rightarrow \infty$ as $x \rightarrow 1$, as suggested in Fig. 4 .

The positive modification at $x=0.1$ is called antishadowing. Although such a positive effect should exist according to the baryon-number, charge, and momentum conservations for a nucleus to cancel the negative modifications of the shadowing and binding, a physics mechanism behind the anti-shadowing is not well investigated. There is a theoretical suggestion on constructive interference in the multiple scattering description [8]. This region is becoming interesting in the sense that the Minerva experiment started producing nuclear modification data in this region in neutrino DIS. The Minerva data seem to indicate large (possibly too large) nuclear modifications in the region $0.05<x<0.2$ [9]. The Fermilab-E772 Drell-Yan experiment indicated no nuclear modification in antiquark distributions, so that the anti-shadowing of $F_{2}^{A}$ should be
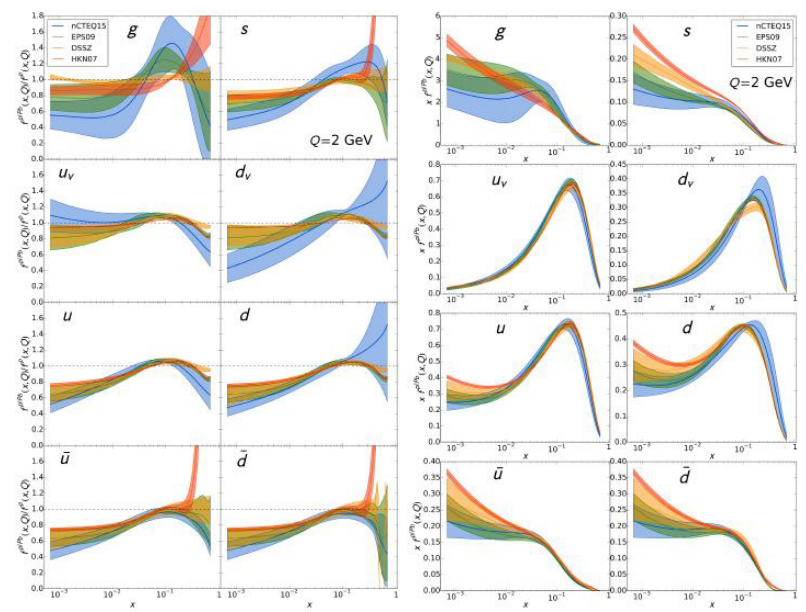

Figure 5. Nuclear modifications and NPDFs in iron [10].

interpreted by valence-quark modifications. Currently, the Fermilab-E906 experiment is in progress, so that new information could be obtained for nuclear antiquark distributions in this region in the near future.

The nuclear parton distribution functions (NPDFs) are determined by analyzing experimental data on nuclear structure functions, Drell-Yan processes, and so on. Typical results are shown in Fig. 5 [10], where the nuclear modifications are shown for each parton distribution on the left-hand side and the NPDFs themselves are shown on the right-hand side at $Q^{2}=2^{2} \mathrm{GeV}^{2}$. Neutrino DIS measurements do not play a major part so far in "directly" finding the nuclear modifications of the PDFs, although measurements have been done for heavy targets such as iron and lead. It is because accurate deuteron measurements do not exist in neutrino reactions, although such data are taken by the ratio form $F_{2}^{A} / F_{2}^{D}$ in charged-lepton reactions as shown in Fig. 4. If a neutrino factory with high-intensity neutrino beam will be realized in future, such data should also be obtained for the deuteron. In determining the nuclear modifications, such ratio data are desirable.

Nuclear corrections are often applied within neutrino experimental collaborations to publish their structure functions of "the nucleon" in final papers. Therefore, the neutrino measurements have been useful in determining the nucleonic PDFs instead of the nuclear PDFs. They are especially valuable for determining the valence-quark distribution through the structure function $F_{3}$ which does not exist in the charged-lepton DIS. Neutrino-induced opposite-sign dimuon events are also important for finding the strange quark distribution in comparison with the light antiquark distributions: $s+\bar{s} \simeq 0.4(\bar{u}+\bar{d})$ at relatively small $Q^{2}$.

In Fig. 5, nCTEQ analysis results are shown in comparison with other distributions. All the analysis results roughly agree within uncertainty bands; however, the gluon modification is not well determined. It could be determined more accurately by using other measurements such as $J / \psi$ production in the ultra-peripheral heavy-ion collisions [11]. In future, the EIC could provide us accurate information on scaling violation of $F_{2}^{A}$ at small $x$ $(<0.004)$ to probe the nuclear gluon shadowing accu- 
rately. In addition, there should be progress in future on flavor dependent nuclear modifications at JLab by the parity-violating DIS and at Fermilab by Drell-Yan processes with nuclear targets.

\section{Gravitational form factors in neutrino scattering through hadron tomography}

We discussed the structure functions and PDFs in Sec. 3 in neutrino DIS. The PDFs indicate distributions of the longitudinal momentum fraction $x$ of partons. Recently, three dimensional structure of the nucleon became a hot topic in hadron physics by including the transverse distributions in

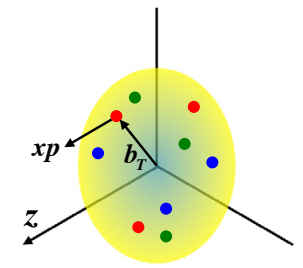

Figure 6. 3D structure. addition to the longitudinal PDFs as illustrated in Fig. 6.

It was motivated by the topic of solving the origin of nucleon spin. According to the original quark model of 1964, the nucleon spin should be interpreted by the combination of three spin-1/2 quark spins. In other words, the nucleon spin should be carried by quarks with $100 \%$ probability. However, polarized charged-lepton DIS measurements indicate that it is a small amount of $20-30 \%$. The nucleon spin is one of fundamental physics quantities, so it is necessary to find its origin. The gluon spin may carry a significant fraction of nucleon spin; however, its contribution has still large uncertainty. It will be clarified by the EIC project. On the other hand, angular momenta of partons could also contribute to the nucleon spin. Their effects should be found by one of three dimensional (3D) structure functions, generalized parton distributions (GPDs), which can be measured by the virtual Compton scattering process on the right-hand side of Fig. 7. Experimental studies are in progress at JLab (Thomas Jefferson National Accelerator Facility) and CERN-COMPASS. The field of 3D structure functions is called hadron tomography. Using this technique, we can determine gravitational sources, namely gravitational masses, pressures, and shear forces, in hadrons in terms of fundamental quark and gluons, and it is also possible in neutrino scattering in principle if its intensity is high enough.

The GPDs are defined matrix elements of non-local vector operators, and their moments are given as

$$
\begin{gathered}
\left.\left(\frac{P^{+}}{2}\right)^{n} \int d x x^{n-1} \int \frac{d y^{-}}{2 \pi} e^{i x P^{+} y^{-} / 2} \bar{q}(-y / 2) \gamma^{+} q(y / 2)\right|_{y^{+}=\vec{y}_{\perp}=0} \\
=\bar{q}(0) \gamma^{+}\left(i \overleftrightarrow{\partial}^{+}\right)^{n-1} q(0) .
\end{gathered}
$$

This operator is the energy-momentum tensor of a quark for $n=2$, and it is a source of gravity, whereas it is the vector-type electromagnetic current for $n=1$ [12]. Therefore, measurements of the GPDs can provide information on the gravitational source within a hadron. The second moments of the GPDs are expressed by gravitational form factors. The electromagnetic form factors of the nucleon have been measured by charged-lepton scattering, and axial form factors are contained in neutrino-scattering cross

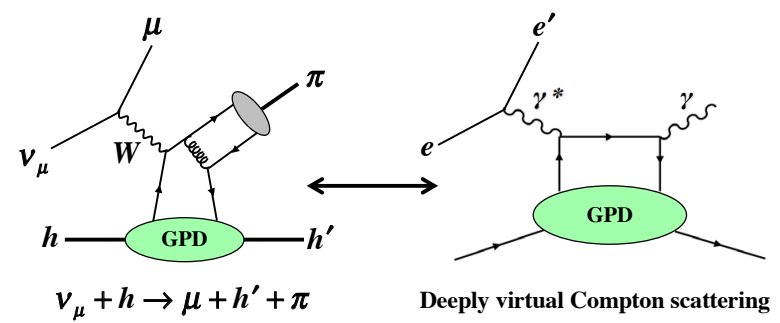

Figure 7. GPDs in $v$ reaction and virtual Compton scattering.

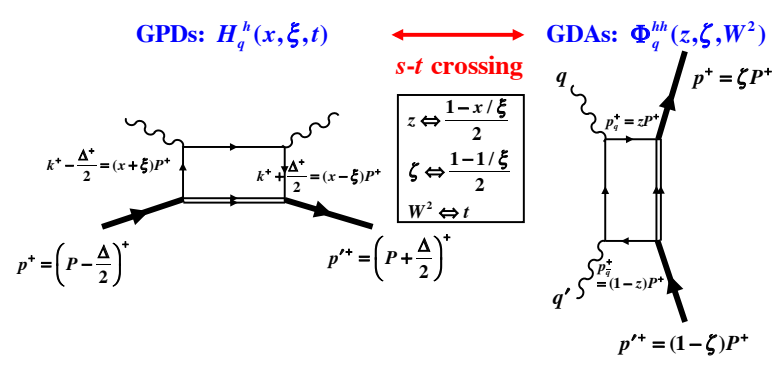

Figure 8. GPDs and GDAs in two-photon process.

sections. There is a way to access the GPDs, for example, by the exclusive pion production in neutrino scattering as shown in Fig. 7 [13]. The pion production processes have been investigated in neutrino reactions; however, they are measured so far at relatively low energies. For measuring the GPDs, namely, for the factorization of the cross section into the soft GPD part and the hard pQCD one, the kinematical condition, $Q^{2} \gg|t|, \Lambda_{Q C D}^{2}$, should be satisfied. In future, if a high-energy and high-intensity neutrino factory will be realized, such measurements could be possible.

There is another way to probe the gravitational form factors by timelike processes as shown in Fig. 8. Instead of the virtual Compton scattering for the GPDs, the 3D structure functions called the generalized distribution amplitudes (GDAs) can be investigated by the two-photon process $\gamma^{*} \gamma \rightarrow h \bar{h}$. Such measurements were first reported by the Belle collaboration in 2016 for $\gamma^{*} \gamma \rightarrow \pi^{0} \pi^{0}$ [14]. The GDAs could be considered as "timelike GPDs", and they are also defined by the same non-local operator of Eq. (6). Therefore, the gravitational form factors can be obtained by analyzing the KEKB measurements.

From the analysis of the two-photon measurements on $\gamma^{*} \gamma \rightarrow \pi^{0} \pi^{0}$ of KEKB, the GPDs of the pion were obtained [12]. There are two gravitational form factors for the spin- 0 pion, and they are calculated by using the determined GDAs. The GDAs are expressed by a number of parameters, which are determined by a $\chi^{2}$ analysis of Belle measurements. The obtained theoretical cross section is shown in Fig. 9 in comparison with typical Belle data. Since it is a timelike process, we should be careful in taking resonances into account in analyzing the Belle data. In fact, the prominent peak is clear for $f_{2}(1270)$, whereas $f_{0}(500)$ and $f_{0}(980)$ are not so obvious in Fig. 9.

Once the quark GDAs $\Phi_{q}^{\pi^{0} \pi^{0}}\left(z, \zeta, W^{2}\right)$ are determined, the form factors are calculated in the following way. The second moment of a GDA is given by the matrix element of the quark energy-momentum tensor $T_{q}^{\mu \nu}$ as 


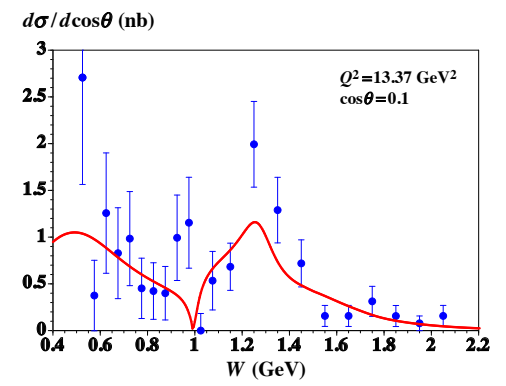

Figure 9. Comparison with Belle cross-section data of $\gamma^{*} \gamma \rightarrow \pi^{0} \pi^{0}$ for determining the GDAs [12].

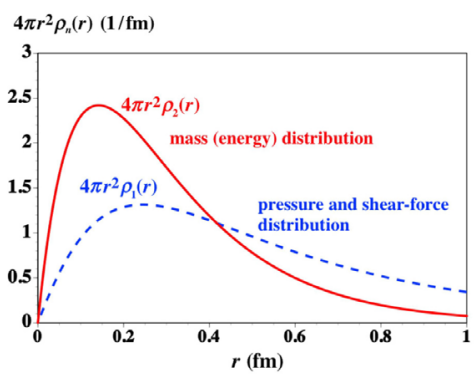

Figure 10. Mass and mechanical distributions obtained from two gravitational form factors $\Theta_{1}$ and $\Theta_{2}$.

$$
\begin{aligned}
\int_{0}^{1} d z & (2 z-1) \Phi_{q}^{\pi^{0} \pi^{0}}\left(z, \zeta, W^{2}\right) \\
= & \frac{2}{\left(P^{+}\right)^{2}}\left\langle\pi^{0}(p) \pi^{0}\left(p^{\prime}\right)\left|T_{q}^{++}(0)\right| 0\right\rangle
\end{aligned}
$$

where $T_{q}^{\mu \nu}$ is defined by $T_{q}^{\mu \nu}(x)=\bar{q}(x) \gamma^{(\mu} \overleftrightarrow{D}^{v)} q(x)$ with the covariant derivative $D^{\mu}=\partial^{\mu}-i g \lambda^{a} A^{a, \mu} / 2$. Here, $g$ is the QCD coupling constant and $\lambda^{a}$ is the SU(3) Gell-Mann matrix. The right-hand side of Eq. (7) can be expressed by the timelike gravitational form factors $\Theta_{1}$ and $\Theta_{2}$ of the pion as

$$
\begin{aligned}
& \left\langle\pi^{0}(p) \pi^{0}\left(p^{\prime}\right)\left|T_{q}^{\mu \nu}(0)\right| 0\right\rangle \\
& \quad=\frac{1}{2}\left[\left(s g^{\mu v}-P^{\mu} P^{v}\right) \Theta_{1, q}(s)+\Delta^{\mu} \Delta^{v} \Theta_{2, q}(s)\right],
\end{aligned}
$$

where $P$ and $\Delta$ are given by the pion momenta as $P=p+p^{\prime}$ and $\Delta=p^{\prime}-p$. In this way, the timelike gravitational form factors are obtained from the Belle measurements. Next, the timelike form factors are converted to spacelike ones by using the dispersion relation, and then they are Fourier transformed to become the spacial mass and mechanical distributions shown in Fig. 10.

From the spacial distributions in Fig. 10, the rootmean-square radii of the pion can be calculated as $\sqrt{\left\langle r^{2}\right\rangle_{\text {mass }}}=0.39 \mathrm{fm}$ and $\sqrt{\left\langle r^{2}\right\rangle_{\text {mech }}}=0.82 \mathrm{fm}$, which correspond to gravitational-mass and mechanical radii. There are some ambiguities in our analysis in assigning phase factors, so that the radius estimates have some uncertainty ranges as: $\sqrt{\left\langle r^{2}\right\rangle_{\text {mass }}}=0.32 \sim 0.39 \mathrm{fm}, \sqrt{\left\langle r^{2}\right\rangle_{\text {mech }}}=$ $0.82 \sim 0.88 \mathrm{fm}[12]$. This is the first result on the gravitational radii from the actual analysis of experimental measurements. The charge radius of the pion has been already measured as $\sqrt{\left\langle r^{2}\right\rangle_{\text {charge }}}=0.672 \pm 0.008 \mathrm{fm}[15]$.

\section{Summary}

High-energy neutrino-nucleon and nucleus scattering processes are interesting and practically important for future precision neutrino oscillation measurements and for finding a possible new phenomenon in ultra-high-energy cosmic neutrino measurements. For application to the neutrino oscillation experiments, we need to combine the neutrino DIS cross section with the ones of other kinematical regions, namely quasi-elastic, resonance, and Regge regions, because neutrino measurements are done at the beam energies form several hundred $\mathrm{MeV}$ to several $\mathrm{GeV}$. On the ultra-high-energy side, the IceCube collaboration started publishing neutrino cross sections in the region of $10^{13}-10^{15} \mathrm{eV}$, so that new phenomenon may be observed in future at the energies beyond the current artificial lepton accelerators. If a high-intensity neutrino factory will be realized in future, neutrino scattering measurements could probe gravitational form factors of hadrons by exclusive processes through the studies of 3D tomography. It is currently a hot topic in the hadron-physics community by using virtual Compton scatting and two-photon processes for measuring the GPDs and GDAs.

\section{Acknowledgments}

Figures 2 and 5 are used with the copyright permission of American Physical Society and authors. The author thanks M. Bustamante for supplying Figure 3.

\section{References}

[1] S. X. Nakamura et al., Rept. Prog. Phys. 80, 056301 (2017).

[2] M. Bustamante and A. Connolly, arXiv:1711.11043; M. G. Aartsen et al., Nature 551, 596 (2017); Erratum 554, 554 (2018).

[3] S. Kumano, arXiv:1809.00115, PoS (DIS2018) 245.

[4] T. De Forest and J. D. Walecka, Adv. Phys. 15, 1 (1966).

[5] M. Miyama and S. Kumano, Comput. Phys. Commun. 94, 185 (1996).

[6] J. A. Formaggio and G. P. Zeller, Rev. Mod. Phys. 84, 1307 (2012).

[7] A. Cooper-Sarkar, P. Mertsch, and S. Sarkar, JHEP 08, 042 (2011).

[8] S. J. Brodsky and H. J. Lu, Phys. Rev. Lett. 64, 1342 (1990); S. J. Brodsky, I. Schmidt, and J.-J. Yang, Phys. Rev. D 70, 116003 (2004).

[9] B. G. Tice et al., Phys. Rev. Lett. 112, 231801 (2014); J. Mousseau et al., Phys. Rev. D 93, 071101(R) (2016).

[10] K. Kovarik et al., Phys. Rev. D 93, 085037 (2016).

[11] V. Guzey et al., Phys. Lett. B 726, 290 (2013).

[12] S. Kumano, Qin-Tao Song, and O. V. Teryaev, Phys. Rev. D 97, 014020 (2018); Erratum in arXiv: 1711.08088 .

[13] B. Pire, L. Szymanowski, and J. Wagner, Phys. Rev. D 95, 114029 (2017).

[14] M. Masuda et al., Phys. Rev. D 93, 032003 (2016).

[15] M. Tanabashi et al., Phys. Rev. D 98, 030001 (2018). 